\title{
Carbazochrome sodium sulfonate is not effective for prevention of post-gastric endoscopic submucosal dissection bleeding: A retrospective study
}

\author{
Keitaro Takahashi ${ }^{1}$ (1) $\cdot$ Takahiro Sasaki $^{1} \cdot$ Nobuhiro Ueno $^{1} \cdot$ Kyoko Uehara $^{1} \cdot$ Yu Kobayashi ${ }^{1} \cdot$ Yuya Sugiyama $^{1}$. \\ Yuki Murakami ${ }^{1}$ - Takehito Kunogi ${ }^{1} \cdot K^{1}$ atsuyoshi Ando ${ }^{1}$ - Shin Kashima ${ }^{1} \cdot K^{1}$ Kentaro Moriichi ${ }^{1} \cdot$ Hiroki Tanabe $^{1}$. \\ Toshikatsu Okumura ${ }^{1} \cdot$ Mikihiro Fujiya $^{1}$
}

Received: 4 October 2021 / Accepted: 21 February 2022 / Published online: 7 March 2022

(c) The Author(s) 2022

\begin{abstract}
Background Carbazochrome sodium sulfonate (CSS) is conventionally administered to prevent post-endoscopic submucosal dissection (ESD) bleeding in many institutions, but research on its preventive efficacy is lacking. Therefore, we investigated the risk of post-ESD bleeding and the preventive efficacy of CSS administration.

Methods We retrospectively reviewed 304 lesions in 259 patients with gastric neoplasms who underwent ESD at Asahikawa Medical University Hospital from 2014 to 2021. In the CSS group, CSS $100 \mathrm{mg} /$ day was intravenously infused with maintenance fluid replacement on postoperative days $0-2$. The risk factors of post-ESD bleeding, including CSS administration, were investigated.

Results The overall rate of post-ESD bleeding was 4.6\% (14/304). The univariate analysis showed that atrial fibrillation (Af), warfarin intake, heparin replacement, and tumor location in the lower third were significant risk factors for increasing the likelihood of postoperative bleeding. In the multivariate analysis, Af (odds ratio [OR] 3.83, 95\% CI 1.02-14.30; $p<0.05$ ), heparin replacement (OR 4.60, 95\% CI 1.02-20.70; $p<0.05$ ), and tumor location in the lower third of the stomach (OR 6.67, 95\% CI 1.43-31.00; $p<0.05$ ) were independent factors for post-ESD bleeding. Post-ESD bleeding was observed in 5.2\% (9/174) of the CSS group and 3.8\% (5/130) of the non-CSS group, with no significant difference between the two groups $(p=0.783$ ). Additionally, CSS was not shown to have preventive effects in groups with higher-risk factors, such as Af diagnosis, warfarin use, heparin replacement, and tumor location in the lower third of the stomach.

Conclusion CSS administration was not effective for the prevention of the post-ESD bleeding in the overall patient population as well as in higher-risk patients. This suggests that the administration of CSS for post-ESD bleeding prevention may need to be reconsidered.
\end{abstract}

Keywords Carbazochrome $\cdot$ Post-operative hemorrhage $\cdot$ Gastric neoplasm $\cdot$ ESD $\cdot$ Prevention

\section{Abbreviations}

CSS Carbazochrome sodium sulfonate

ESD Endoscopic submucosal dissection

OR Odds ratio; 95\% CI: 95\% confidence interval

P-CAB Potassium-competitive acid blocker

Keitaro Takahashi

ktakaha@asahikawa-med.ac.jp

1 Gastroenterology and Endoscopy, Division of Metabolism and Biosystemic Science, Gastroenterology, and Hematology/Oncology, Department of Medicine, Asahikawa Medical University, 2-1 Midorigaoka-higashi, Asahikawa, Hokkaido 078-8510, Japan
Af Atrial fibrillation

PPI Proton pump inhibitor

Endoscopic submucosal dissection (ESD) has been widely used for treating gastric neoplasms. ESD provides a high en bloc resection rate and overall good prognosis, although one of its major complications is postoperative bleeding [1]. The Japan Gastroenterological Endoscopy Society recommends use of hemostatic forceps to coagulate remnant vessels as well as the administration of a proton pump inhibitor (PPI) or an H2-histamine receptor antagonist to prevent post-ESD bleeding [2]. However, postoperative bleeding continues to occur in about 4-8\% of gastric ESDs [3]. Various preventive 
methods, such as the polyglycolic acid shielding method, ulcer base closure, and use of polysaccharide hemostatic powder, have been implemented but have not significantly prevented post-ESD bleeding [4-6].

Carbazochrome sodium sulfonate (CSS) is a hemostatic agent that reduces capillary permeability and increases capillary resistance, resulting in shortened bleeding time $[7,8]$. CSS has been used to treat bleeding of the gastrointestinal and respiratory tracts. The hemostatic effect of CSS has been shown in instances of hereditary hemorrhagic telangiectasia and total knee arthroplasty; however, CSS has not demonstrated hemostatic effects in instances of colonic diverticular bleeding [7-9]. Therefore, research regarding the hemostatic effects of CSS is currently controversial, especially in cases of gastrointestinal bleeding.

In the perioperative period of gastric ESD, CSS is conventionally administered to prevent post-ESD bleeding in many institutions, but there have been no reports verifying its preventive efficacy. Herein, we investigated post-ESD bleeding and the preventive efficacy of CSS administration.

\section{Materials and methods}

\section{Study patients}

We retrospectively reviewed a total of 328 consecutive lesions in 283 patients with gastric neoplasms who underwent ESD at Asahikawa Medical University Hospital from November 2014 to April 2021. We excluded five cases with non-neoplastic lesions, five cases with a gastric remnant, and fourteen cases with ESD discontinuation. Finally, 259 patients with a total of 304 gastric neoplasms were enrolled in the study. This study was approved by the institutional ethics committee of Asahikawa Medical University.

\section{Pre-ESD management of antithrombotic agents}

In patients undergoing antithrombotic treatment, antithrombotic agents were withdrawn based on our hospital regulations and guidelines of the Japan Gastroenterological Endoscopy Society for patients undergoing antithrombotic treatment $[10,11]$. Use of aspirin was discontinued for 3-5 days prior to the ESD procedure. Use of thienopyridine derivatives was halted 5-7 days in advance of the procedure. Warfarin was withdrawn for 3-4 days prior to the ESD procedure, and heparin replacement was withheld until the morning of the procedure. Direct oral anticoagulants (DOAC) and other antiplatelet agents were withdrawn on the morning of the procedure.

\section{ESD procedures}

ESD was carried out by endoscopists at Asahikawa Medical University. A single-channel upper gastrointestinal endoscope (GIF-Q260J; Olympus Medical Systems, Tokyo, Japan) was used with a high-frequency generator (VIO-300D; Erbe Elektromedizin GmbH, Tübingen, Germany). The endoscopists selected an electrosurgical knife from FlushKnife BT-S (DK2620J; Fujifilm, Tokyo, Japan), a DualKnifeJ (KD-655L; Olympus Medical Systems, Tokyo, Japan), or an IT knife 2 (KD-610L; Olympus Medical Systems, Tokyo, Japan). Circumferential markings were made outside the tumor margin under the magnifying endoscopy with narrow-band imaging. Hyaluronic acid solution (Mucoup; Boston Scientific, Tokyo, Japan or Ksmart; Olympus Medical Systems, Tokyo, Japan) was injected into the submucosal layer to lift the surrounding mucosa. The mucosal incision was completed around the markings. Submucosal dissection was then initiated from the proximal side to the distal side and en bloc resection was performed. Immediately after ESD, a coagrasper (FD412LR; Olympus Medical Systems, Tokyo, Japan) was utilized to discontinue hemorrhage from exposed blood vessels on the artificial ulcer. Ulcer base closure was not performed in any cases.

\section{Post-ESD clinical pathway}

A clinical pathway for our institution is shown in Fig. 1. After ESD, the patients maintained fasting conditions, and maintenance fluid replacement was administered. In our clinical pathway from November 2014 to March 2019, CSS was intravenously infused at $100 \mathrm{mg}$ /day with maintenance fluid replacement on postoperative days 0-2. In April 2019, the clinical pathway was reviewed, and the infusion of CSS was stopped. Maintenance fluid replacement was utilized without CSS from April 2019 to April 2021. Regarding the gastric acid-suppressing agents, omeprazole sodium $40 \mathrm{mg} /$ day (omeprazole; Nichi-Iko Pharmaceutical Company, Toyama, Japan) was injected on postoperative days $0-1$, and either esomeprazole magnesium $20 \mathrm{mg} /$ day (Nexium; Daiichi Sankyo Company, Tokyo, Japan) or vonoprazan fumarate $20 \mathrm{mg} /$ day (Takecab; Takeda Pharmaceutical Company, Tokyo, Japan) was administered on and after postoperative day 2 . The scheduled follow-up endoscopy was not performed in our clinical pathway. Instead, blood tests and physical examinations were completed on postoperative day 1 , and an emergency endoscopy was performed on patients with hematemesis/melena or in patients with a decline in hemoglobin levels by $\geq 2 \mathrm{mg} / \mathrm{dL}$. In cases where nothing abnormal was detected from the blood tests or physical 


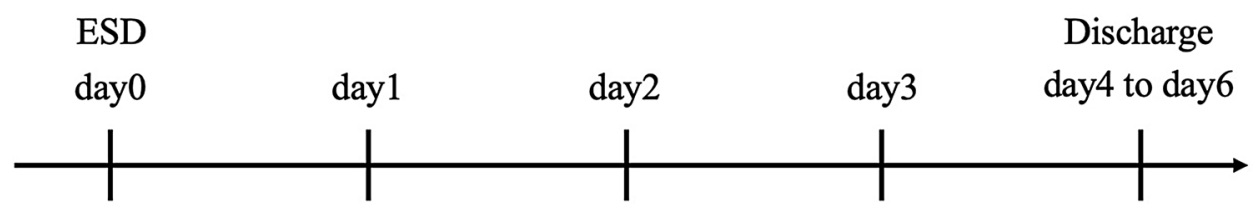

\begin{tabular}{l}
\begin{tabular}{|l|}
\hline - CSS 100 mg/day (from Nov. 2014 to Mar. 2019) \\
• Non-CSS (from Apr. 2019 to Apr. 2021) \\
\hline Maintenance fluid replacement \\
\hline Intravenous PPI \\
\hline Fasting \\
\hline
\end{tabular} \\
\hline
\end{tabular}

Fig. 1 Post-endoscopic submucosal dissection clinical pathway. After endoscopic submucosal dissection (ESD), the patients were under fasting conditions, and maintenance fluid replacements were administered. Carbazochrome sodium sulfonate (CSS) (100 mg/day) was intravenously infused with maintenance fluid replacement on postoperative days 0-2 from November 2014 to March 2019. Maintenance fluid replacement without CSS was utilized from April 2019 to April

examinations, antithrombotic agents were resumed on the day after the ESD procedure, and oral intake was reintroduced on postoperative day 2 . The patients were discharged from our hospital on postoperative days 4-6.

\section{Post-ESD management for bleeding}

Post-ESD bleeding is defined as hematemesis/melena required for endoscopic hemostasis or a decline in hemoglobin levels by $\geq 2 \mathrm{mg} / \mathrm{dL}$. When post-ESD bleeding occurred, we performed emergency endoscopic hemostasis. After endoscopic hemostasis, the timing of antithrombotic agent resumption was determined by the endoscopists.

\section{Statistical analyses}

All statistical analyses were performed using the R Project for Statistical Computing version 4.0.5 software program. Continuous variables were compared using Student's $t$ test, and nominal scale data were compared using Fisher's exact probability test. Odds ratios (ORs) and $95 \%$ confidence intervals $(95 \%$ CIs) were calculated to evaluate the strength of the influence of each individual variable. Selected variables with $p$-values $<0.05$ in univariate analysis were included in the multivariate analysis. $p$-values of $<0.05$ were considered to indicate statistical significance.
2021. Regarding the gastric acid-suppressing agents, intravenous proton pump inhibitor (PPI) was injected on postoperative days 0-1 and then the PPI or potassium-competitive acid blocker (P-CAB) was administered on and after postoperative day 2 . Oral intake was reintroduced on postoperative day 2 . The patients were discharged from our hospital on postoperative days 4-6.

\section{Results}

\section{Risk factors and rate of post-ESD bleeding}

The overall rate of post-ESD bleeding was 4.6\% (14/304). Regarding the risk of post-ESD bleeding, Table 1 shows the clinicopathological features of the post-ESD bleeding and non-bleeding groups. In the post-ESD bleeding group, characteristics including Af, warfarin intake, heparin replacement, and tumor location at the lower third of the stomach were significantly higher compared to the non-bleeding group $(p<0.05)$. Other factors (age, gender, antithrombotic agent use, potassium-competitive acid blocker (P-CAB) use, macroscopic type, histology, ulceration, depth of invasion, size of the resected specimen, tumor size, resection time, en bloc resection, R0 resection, and intraoperative perforation) did not differ between the post-ESD bleeding and non-bleeding groups.

The results of the univariate and multivariate analyses for factors of post-ESD bleeding are summarized in Table 2. The univariate analysis showed significant risk factors and characteristics as follows: Af (OR 5.35, 95\% confidence interval (95\% CI) 1.12-20.75; $p=0.018)$, warfarin intake (OR 15.13, 95\% CI 2.09-90.18; $p=0.004$ ), heparin replacement (OR 6.24, 95\% CI 0.99-28.35; $p=0.026$ ), and tumor location at the lower third of the 
Table 1 Clinicopathological features of the post-ESD bleeding and non-bleeding groups
Post-ESD bleeding, Non-bleeding, $n=290$

$p$ value $n=14$

\begin{tabular}{|c|c|c|c|}
\hline Age (years, mean $\pm S D$ ) & $71.1(4.3)$ & $73.4(8.3)$ & 0.309 \\
\hline Gender, $n(\%)$ & & & 1 \\
\hline Male & $10(71.4)$ & $172(70.2)$ & \\
\hline Female & $4(28.6)$ & $73(29.8)$ & \\
\hline \multicolumn{4}{|l|}{ Co-morbidity } \\
\hline Atrial fibrillation & $4(28.6)$ & $20(6.9)$ & 0.018 \\
\hline Ischemic heart disease & $0(0)$ & $12(4.1)$ & 1 \\
\hline Cerebrovascular disease & $0(0)$ & $22(7.6)$ & 0.61 \\
\hline Chronic kidney disease on HD & $0(0)$ & $4(1.4)$ & 1 \\
\hline \multicolumn{4}{|l|}{ Antithrombotic therapy, $n(\%)$} \\
\hline Aspirin & $0(0)$ & $24(8.3)$ & 0.612 \\
\hline Thienopyridine derivatives & $0(0)$ & $20(6.9)$ & 0.610 \\
\hline Cilostazol & $0(0)$ & $10(3.4)$ & 1 \\
\hline Warfarin & $3(21.4)$ & $5(1.7)$ & 0.004 \\
\hline DOAC & $2(14.3)$ & $24(8.3)$ & 0.340 \\
\hline Others & $1(7.1)$ & $23(7.9)$ & 1 \\
\hline Antithrombotic agent use, $n(\%)$ & $6(42.9)$ & $86(29.7)$ & 0.371 \\
\hline Single & $6(42.9)$ & $66(22.8)$ & 0.106 \\
\hline Doublet & $0(0)$ & $15(5.2)$ & 1 \\
\hline Triplet & $0(0)$ & $3(1)$ & 1 \\
\hline Heparin replacement & $3(21.4)$ & $12(4.1)$ & 0.026 \\
\hline P-CAB & $2(14.3)$ & $36(12.4)$ & 0.690 \\
\hline \multicolumn{4}{|l|}{ Tumor location, $n(\%)$} \\
\hline Lower third & $12(85.7)$ & $132(45.5)$ & 0.004 \\
\hline Middle third & $2(14.3)$ & $101(34.8)$ & 0.151 \\
\hline Upper third & $0(0)$ & $56(19.3)$ & 0.081 \\
\hline \multicolumn{4}{|l|}{ Macroscopic type, $n(\%)$} \\
\hline Elevated & $6(42.9)$ & $131(45.2)$ & 1 \\
\hline Flat/depressed & $8(57.1)$ & $130(44.8)$ & 0.418 \\
\hline mixed & $0(0)$ & $29(10)$ & 0.376 \\
\hline \multicolumn{4}{|l|}{ Histology } \\
\hline Adenoma & $4(28.6)$ & $54(18.6)$ & 0.316 \\
\hline Differentiated type & $10(71.4)$ & $223(76.9)$ & 0.746 \\
\hline Undifferentiated type & $0(0)$ & $13(4.5)$ & 1 \\
\hline $\mathrm{UL}+, n(\%)$ & $1(7.1)$ & $22(7.6)$ & 1 \\
\hline \multicolumn{4}{|l|}{ Depth } \\
\hline $\mathrm{SM} 2, n(\%)$ & $0(0)$ & $16(5.5)$ & 1 \\
\hline Resection size, mm (mean \pm SD) & $39.3(16.9)$ & $36.9(12.5)$ & 0.494 \\
\hline Resection size > $30 \mathrm{~mm}, n(\%)$ & $9(64.3)$ & $188(64.8)$ & 1 \\
\hline Tumor size, mm (mean $\pm \mathrm{SD})$ & $19.1(10.7)$ & $16.5(11.2)$ & 0.396 \\
\hline Resection time, minutes (mean \pm SD) & $106.6(66.4)$ & $103.4(71.6)$ & 0.866 \\
\hline Resection time $>100 \min , n(\%)$ & $8(57.1)$ & $117(40.3)$ & 0.268 \\
\hline En bloc resection, $n(\%)$ & $14(100)$ & $281(96.9)$ & 1 \\
\hline $\mathrm{R} 0$ resection, $n(\%)$ & $14(100)$ & $272(93.8)$ & 1 \\
\hline Intraoperative perforation, $n(\%)$ & $0(0)$ & $5(1.7)$ & 1 \\
\hline
\end{tabular}

$H D$ hemodialysis, $D O A C$ direct oral anticoagulants, $P-C A B$ potassium-competitive acid blocker, $U L$ ulceration, $S D$ standard deviation 
Table 2 Univariate and multivariate analyses for factors of post-ESD bleeding

\begin{tabular}{|c|c|c|c|c|c|c|}
\hline & \multicolumn{3}{|c|}{ Univariate analysis } & \multicolumn{3}{|c|}{ Multivariate analysis } \\
\hline & OR & $95 \%$ CI & $p$ value & OR & $95 \% \mathrm{CI}$ & $p$ value \\
\hline Atrial fibrillation & 5.35 & $1.12-20.75$ & 0.018 & 3.83 & $1.02-14.30$ & 0.046 \\
\hline Warfarin & 15.13 & $2.09-90.18$ & 0.004 & - & - & - \\
\hline Heparin replacement & 6.24 & $0.99-28.35$ & 0.026 & 4.60 & $1.02-20.70$ & 0.046 \\
\hline Lower third & 7.14 & $1.55-66.93$ & 0.004 & 6.67 & $1.43-31.00$ & 0.016 \\
\hline
\end{tabular}

stomach (OR 7.14, 95\% CI 1.55-66.93; $p=0.004)$. In the multivariate analysis, warfarin administration and heparin replacement showed high values of variance inflation factors. Therefore, warfarin administration was excluded from the analysis. As a result, Af (OR 3.83, 95\% CI 1.02-14.30; $p=0.046)$, heparin replacement (OR $4.60,95 \%$ CI $1.02-20.70 ; p=0.046)$, and location of the tumor in the lower third of the stomach (OR 6.67, 95\% CI $1.43-31.00 ; p=0.016$ ) were independent factors for post-ESD bleeding.

With regard to the onset of post-ESD bleeding, 4 patients were in early-phase bleeding within $24 \mathrm{~h}$ of the procedure and 10 patients presented with late-phase bleeding after $24 \mathrm{~h}$. The mean resection size was significantly higher in the early-phase bleeding group compared to the non-bleeding group $(52.3 \pm 26.1 \mathrm{~mm}$ vs $36.8 \pm 26.1 \mathrm{~mm} ; p<0.05)$. The other factors did not differ between the early-phase bleeding group and the non-bleeding group. In the analysis of latephase bleeding, the risk factors were the same as the overall post-ESD bleeding group.

\section{The influence of CSS administration for post-ESD bleeding}

Regarding clinicopathological features, in the CSS group, the adenoma size, resection time, and rate of resection time over 100 min were significantly higher compared to the nonCSS group $(p<0.05)$ (Table 3). The differentiated type was significantly lower than non-CSS. Other factors were not significantly different between the groups. Overall, post-ESD bleeding was observed in 5.2\% (9/174) of the CSS group and $3.8 \%(5 / 130)$ of the non-CSS group, with no significant difference between the two groups $(p=0.783)$ (Table 4$)$. The median onset of post-ESD bleeding was day $2(0-9)$ in the CSS group and day $0(0-2)$ in the non-CSS group, which did not show a significant difference $(p=0.104)$. Among patients with Af, post-ESD bleeding was observed in 3 patients of the CSS group (21.4\%), whereas in the non-CSS group, it was observed in 1 patient (10.0\%). Among patients taking warfarin, post-ESD bleeding was observed in 3 patients of the CSS group (60.0\%), whereas it was not observed in the non-CSS group (0\%). Similarly, for patients with heparin replacement, post-ESD bleeding was observed in 3 patients of the CSS group (30.0\%), while it was not observed in the non-CSS group (0\%). Among patients with the tumor location in the lower third of the stomach, post-ESD bleeding was observed in 8 patients of the CSS group (10.7\%), while it was observed in 4 patients $(5.8 \%)$ in the non-CSS group. Thus, these data suggest that CSS administration was not effective for the prevention of post-ESD bleeding in the general population or in high-risk patients who were reviewed for this study.

The chronological trend of post-ESD bleeding in the patients with CSS was analyzed before and after July 2017 because the guidelines for gastroenterological endoscopy in patients undergoing antithrombotic treatment was revised in Japan in July 2017 [11]. The rate of post-ESD bleeding was 6.7\% (6/90) before July 2017 and 3.5\% (3/84) after July 2017, which did not show a significant difference $(p=0.499)$. As a result, the preventive efficacy of CSS administration did not significantly change in terms of the chronological trend.

\section{Discussion}

This is the first report to show the inefficacy of CSS administration for the prevention of the post-ESD bleeding, suggesting little merit of CSS administration for the prevention of the post-ESD bleeding.

In this study, the overall rate of post-ESD bleeding was 4.6\%. The univariate analysis showed that Af, warfarin intake, heparin replacement, and tumor location in the lower third of the stomach were risk factors in the post-ESD bleeding group. The multivariate analysis showed that Af, heparin replacement, and tumor location in the lower third of the stomach were independent factors for post-ESD bleeding. It is well known that antithrombotic agents, including warfarin and heparin replacement, are risk factors for post-ESD bleeding [12]. Additionally, the lower third of the stomach is influenced by gastric peristalsis of antrum and bile or digestive enzyme reflux from the duodenum [13], so the location of the tumor in the lower third of the stomach was a highrisk factor for post-ESD bleeding. Then, we analyzed the rate of post-ESD bleeding among subgroups of patients who received CSS with such risk factors. The results showed that CSS administration was ineffective for preventing post-ESD bleeding, clearly indicating that CSS administration is not 
Table 3 Clinicopathological features of the CSS and nonCSS groups

\begin{tabular}{|c|c|c|c|}
\hline & $\mathrm{CSS}, n=174$ & Non-CSS, $n=130$ & $p$ value \\
\hline Age, mean (SD) & $72.6(8.6)$ & $74.1(7.6)$ & 0.110 \\
\hline Gender, $n(\%)$ & & & 1 \\
\hline Male & $103(70.1)$ & $94(72.3)$ & \\
\hline Female & $44(29.9)$ & $36(27.7)$ & \\
\hline \multicolumn{4}{|l|}{ Co-morbidity (/lesion) } \\
\hline Atrial fibrillation & $14(8.0)$ & $10(7.7)$ & 1 \\
\hline Ischemic heart disease & $5(2.9)$ & $7(5.4)$ & 0.373 \\
\hline Cerebrovascular disease & $11(6.3)$ & $11(8.5)$ & 0.508 \\
\hline Chronic kidney disease on HD & $3(1.7)$ & $1(0.8)$ & 0.638 \\
\hline Antithrombotic agent therapy, $n(\%)$ & (/lesion) & & \\
\hline Aspirin & $12(6.9)$ & $12(7.1)$ & 0.521 \\
\hline Thienopyridine derivatives & $12(6.9)$ & $8(6.2)$ & 1 \\
\hline Cilostazol & $8(4.6)$ & $2(1.5)$ & 0.198 \\
\hline Warfarin & $5(2.9)$ & $3(2.3)$ & 1 \\
\hline DOAC & $18(10.3)$ & $8(6.2)$ & 0.220 \\
\hline others & $11(6.3)$ & $13(10.0)$ & 0.284 \\
\hline Antithrombotic agent use, $n(\%)$ & $53(30.5)$ & $39(30.0)$ & 1 \\
\hline Single & $41(23.6)$ & $31(23.8)$ & 1 \\
\hline Doublet & $9(5.2)$ & $6(4.6)$ & 1 \\
\hline Triplet & $2(1.1)$ & $1(0.8)$ & 1 \\
\hline Heparin replacement & $10(5.7)$ & $5(3.8)$ & 0.595 \\
\hline $\mathrm{P}-\mathrm{CAB}$ & $18(10.3)$ & $20(15.4)$ & 0.221 \\
\hline \multicolumn{4}{|l|}{ Tumor location, $n(\%)$} \\
\hline Lower third & $75(43.1)$ & $69(53.1)$ & 0.104 \\
\hline Middle third & $65(37.4)$ & $38(29.2)$ & 0.144 \\
\hline Upper third & $34(19.5)$ & $22(16.9)$ & 0.654 \\
\hline \multicolumn{4}{|l|}{ Macroscopic type, $n(\%)$} \\
\hline Elevated & $72(41.4)$ & $65(50.0)$ & 0.162 \\
\hline Flat/depressed & $81(46.6)$ & $57(43.8)$ & 0.644 \\
\hline Mixed & $21(12.1)$ & $8(6.2)$ & 0.113 \\
\hline \multicolumn{4}{|l|}{ Histology } \\
\hline Adenoma & $44(25.3)$ & $14(10.8)$ & 0.002 \\
\hline Differentiated type & $123(70.7)$ & $110(84.6)$ & 0.006 \\
\hline Undifferentiated type & $7(4.0)$ & $6(4.6)$ & 0.784 \\
\hline $\mathrm{UL}(+), n(\%)$ & $15(8.6)$ & $8(6.2)$ & 0.513 \\
\hline \multicolumn{4}{|l|}{ Depth } \\
\hline $\mathrm{SM} 2, n(\%)$ & $8(4.6)$ & $8(6.2)$ & 0.609 \\
\hline Resection size, $m m($ mean $\pm S D)$ & $37.2(12.9)$ & $36.8(12.4)$ & 0.808 \\
\hline Resection size > $30 \mathrm{~mm}, n(\%)$ & $115(66.1)$ & $82(63.1)$ & 0.628 \\
\hline Tumor size, mm(mean $\pm \mathrm{SD})$ & $17.4(11.6)$ & $15.6(10.7)$ & 0.177 \\
\hline Resection time, minutes $($ mean $\pm S D)$ & $112.0(74.5)$ & $92.1(65.2)$ & 0.015 \\
\hline Resection time $>100 \min , n(\%)$ & $81(46.6)$ & $44(33.8)$ & 0.034 \\
\hline En bloc resection, $n(\%)$ & $167(96.0)$ & $128(98.5)$ & 0.309 \\
\hline $\mathrm{R} 0$ resection, $n(\%)$ & $161(92.5)$ & $125(96.2)$ & 0.225 \\
\hline Intraoperative perforation, $n(\%)$ & $5(2.9)$ & $0(0)$ & 0.074 \\
\hline
\end{tabular}

recommended for preventing post-ESD bleeding, even in the high-risk group.

In previous research, CSS administration has been shown to have a hemostatic effect in the total knee arthroplasty without impacting the fibrinolysis system [7, 14]. However, these orthopedic findings were based on excessive bleeding within 1 or 2 days post-operation. In gastric ESD, uncontrolled hemorrhage is rarely experienced due to advances 
Table 4 Post-ESD bleeding of the CSS and non-CSS groups

\begin{tabular}{llll}
\hline Post-ESD bleeding & CSS & Non-CSS & $p$ value \\
\hline Overall, $n(\%)$ & $9 / 174(5.2)$ & $5 / 130(3.8)$ & 0.783 \\
Median onset of post- & $2(0-9)$ & $0(0-2)$ & 0.104 \\
$\quad$ ESD bleeding, day & & & \\
Atrial fibrillation & $3 / 14(21.4)$ & $1 / 10(10.0)$ & 0.615 \\
Warfarin & $3 / 5(60.0)$ & $0 / 3(0)$ & 0.196 \\
Heparin replacement & $3 / 10(10.0)$ & $0 / 5(0)$ & 0.505 \\
Lower third & $8 / 75(10.7)$ & $4 / 69(5.8)$ & 0.372 \\
\hline
\end{tabular}

in bleeding-preventive techniques and devices. The average value of decreased hemoglobin level was reported to be around $1.0 \mathrm{~g} / \mathrm{dL}$ after ESD [15]. Therefore, the limited amount of blood loss during ESD might weaken the hemostatic effect of CSS. In addition, CSS is thought to approach the capillary vessels and provide a hemostatic effect [8]. Because exposed blood vessels on the artificial ulcer are usually ablated during ESD, CSS might not exert the hemostatic effect in such situations.

This study was associated with several limitations. First, this was a retrospective and a single-center study; however, the rate of post-ESD bleeding is similar to other reports [2]. Second, the sample size of patients with post-ESD bleeding was relatively small, even though the CSS group and nonCSS group included more than 130 patients each. Third, a background of the CSS group and the non-CSS group showed a significant difference in histology type, resection time, and rate of resection time over $100 \mathrm{~min}$, although our analysis revealed that these factors were not related to the risk of the post-ESD bleeding (Tables 1 and 2).

In conclusion, CSS administration was not effective for the prevention of post-ESD bleeding in both the general population and in the higher-risk patients analyzed for this study. This suggests that the administration of CSS for post-ESD bleeding prevention may need to be reconsidered.

Acknowledgements We thank Takashi Teramoto, associated professor of applied mathematics, for assistance with the statistical analysis and Misaki Katakura for assistance with the data input.

Author contributions $\mathrm{KT}$ and MF conducted the study and wrote the initial draft of the manuscript. KT, TS, KU, YK, YS, YM, TK, and NU performed the ESD procedures. NU and SK contributed to the analysis and interpretation of the data. KM, HT, and TO have contributed to data collection and interpretation and have also critically reviewed the manuscript. All authors approved the final version of the manuscript and agree to be accountable for all aspects of the work in ensuring that questions related to the accuracy or integrity of any part of the work are appropriately investigated and resolved.

Funding The authors have received no funding.

Data availability The datasets supporting the conclusions of this article can be made available upon request.

\section{Declarations}

Disclosures Mikihiro Fujiya received lecture fees from Olympus Corporation and Fujifilm Corporation. Keitaro Takahashi, Takahiro Sasaki, Nobuhiro Ueno, Kyoko Uehara, Yu Kobayashi, Yuya Sugiyama, Yuki Murakami, Takehito Kunogi, Katsuyoshi Ando, Shin Kashima, Kentaro Moriichi, Hiroki Tanabe, and Toshikatsu Okumura have no conflicts of interest or financial ties to disclose.

\section{Ethical approval and consent to participate}

The study was reviewed and approved by the Institutional Review Boards of Asahikawa Medical University. We used the patient optout consent method for participation in this study. We retrospectively reviewed anonymized clinical data after each patient received standard management. Individuals cannot be identified based on the data presented.

Consent for publication Not applicable.

Open Access This article is licensed under a Creative Commons Attribution 4.0 International License, which permits use, sharing, adaptation, distribution and reproduction in any medium or format, as long as you give appropriate credit to the original author(s) and the source, provide a link to the Creative Commons licence, and indicate if changes were made. The images or other third party material in this article are included in the article's Creative Commons licence, unless indicated otherwise in a credit line to the material. If material is not included in the article's Creative Commons licence and your intended use is not permitted by statutory regulation or exceeds the permitted use, you will need to obtain permission directly from the copyright holder. To view a copy of this licence, visit http://creativecommons.org/licenses/by/4.0/.

\section{References}

1. Yang CH, Qiu Y, Li X, Shi RH (2020) Bleeding after endoscopic submucosal dissection of gastric lesions. J Dig Dis 21:139-146. https://doi.org/10.1111/1751-2980.12850

2. Ono H, Yao K, Fujishiro M, Oda I, Nimura S, Yahagi N, Iishi H, Oka M, Ajioka Y, Ichinose M, Matsui T (2016) Guidelines for endoscopic submucosal dissection and endoscopic mucosal resection for early gastric cancer. Dig Endosc 28:3-15. https:// doi.org/10.1111/den. 12518

3. Hatta W, Tsuji Y, Yoshio T, Kakushima N, Hoteya S, Doyama H, Nagami Y, Hikichi T, Kobayashi M, Morita Y, Sumiyoshi T, Iguchi M, Tomida H, Inoue T, Koike T, Mikami T, Hasatani K, Nishikawa J, Matsumura T, Nebiki H, Nakamatsu D, Ohnita K, Suzuki H, Ueyama H, Hayashi Y, Sugimoto M, Yamaguchi S, Michida T, Yada T, Asahina Y, Narasaka T, Kuribasyashi S, Kiyotoki S, Mabe K, Nakamura T, Nakaya N, Fujishiro M, Masamune A (2021) Prediction model of bleeding after endoscopic submucosal dissection for early gastric cancer: BEST-J score. Gut 70:476-484. https://doi.org/10.1136/gutjnl-2019-319926

4. Kataoka Y, Tsuji Y, Hirasawa K, Takimoto K, Wada T, Mochizuki S, Ohata K, Sakaguchi Y, Niimi K, Ono S, Kodashima S, Yamamichi N, Fujishiro M, Koike K (2019) Endoscopic tissue shielding to prevent bleeding after endoscopic submucosal dissection: a prospective multicenter randomized controlled trial. Endoscopy 51:619-627. https://doi.org/10.1055/a-0860-5280

5. Ego M, Abe S, Nonaka S, Suzuki H, Yoshinaga S, Oda I, Saito Y (2021) Endoscopic closure utilizing endoloop and endoclips after gastric endoscopic submucosal dissection for patients on 
antithrombotic therapy. Dig Dis Sci 66:2336-2344. https://doi. org/10.1007/s10620-020-06508-8

6. Jung DH, Moon HS, Park CH, Park JC (2021) Polysaccharide hemostatic powder to prevent bleeding after endoscopic submucosal dissection in high risk patients: a randomized controlled trial. Endoscopy 53:994-1002. https://doi.org/10. 1055/a-1312-9420

7. Luo Y, Zhao X, Releken Y, Yang Z, Pei F, Kang P (2020) Hemostatic and anti-inflammatory effects of carbazochrome sodium sulfonate in patients undergoing total knee arthroplasty: a randomized controlled trial. J Arthroplast 35:61-68. https://doi.org/ 10.1016/j.arth.2019.07.045

8. Miyamoto Y, Ohbe H, Ishimaru M, Matsui H, Fushimi K, Yasunaga $H$ (2020) The effect of carbazochrome sodium sulfonate in patients with colonic diverticular bleeding: propensity score matching analyses using a nationwide inpatient database. Intern Med 59:1789-1794. https://doi.org/10.2169/internalmedicine. 4308-19

9. Passali GC, De Corso E, Bastanza G, Di Gennaro L, HHT Gemelli Study Group (2015) An old drug for a new application: carbazochrome-sodium-sulfonate in HHT. J Clin Pharmacol 55:601602. https://doi.org/10.1002/jcph.452

10. Fujimoto K, Fujishiro M, Kato M, Higuchi K, Iwakiri R, Sakamoto C, Uchiyama S, Kashiwagi A, Ogawa H, Murakami K, Mine T, Yoshino J, Kinoshita Y, Ichinose M, Matsui T, Japan Gastroenterological Endoscopy Society (2014) Guidelines for gastroenterological endoscopy in patients undergoing antithrombotic treatment. Dig Endosc 26:1-14. https://doi.org/10.1111/den.12183

11. Kato M, Uedo N, Hokimoto S, Ieko M, Higuchi K, Murakami K, Fujimoto K (2018) Guidelines for gastroenterological endoscopy in patients undergoing antithrombotic treatment: 2017 appendix on anticoagulants including direct oral anticoagulants. Dig Endosc 30:433-440. https://doi.org/10.1111/den.13184

12. Kataoka Y, Tsuji Y, Sakaguchi Y, Minatsuki C, Asada-Hirayama I, Niimi K, Ono S, Kodashima S, Yamamichi N, Fujishiro M, Koike $\mathrm{K}$ (2016) Bleeding after endoscopic submucosal dissection: risk factors and preventive methods. World J Gastroenterol 22:5927. https://doi.org/10.3748/wjg.v22.i26.5927

13. Nam HS, Choi CW, Kim SJ, Kim HW, Kang DH, Park SB, Ryu DG (2019) Risk factors for delayed bleeding by onset time after endoscopic submucosal dissection for gastric neoplasm. Sci Rep 9:2674. https://doi.org/10.1038/s41598-019-39381-1

14. Onodera T, Majima T, Sawaguchi N, Kasahara Y, Ishigaki T, Minami A (2012) Risk of deep venous thrombosis in drain clamping with tranexamic acid and carbazochrome sodium sulfonate hydrate in total knee arthroplasty. J Arthroplast 27:105-108. https://doi.org/10.1016/j.arth.2011.02.004

15. Kakushima N, Ono H, Takizawa K, Tanaka M, Kawata N, Yoshida M, Murai K, Yabuuchi Y, Kishida Y, Ito S, Imai K, Hotta K, Ishiwatari H, Matsubayashi H (2019) Incidence of delayed bleeding among patients continuing antithrombotics during gastric endoscopic submucosal dissection. Intern Med 58:2759-2766. https:// doi.org/10.2169/internalmedicine.2754-19

Publisher's Note Springer Nature remains neutral with regard to jurisdictional claims in published maps and institutional affiliations. 